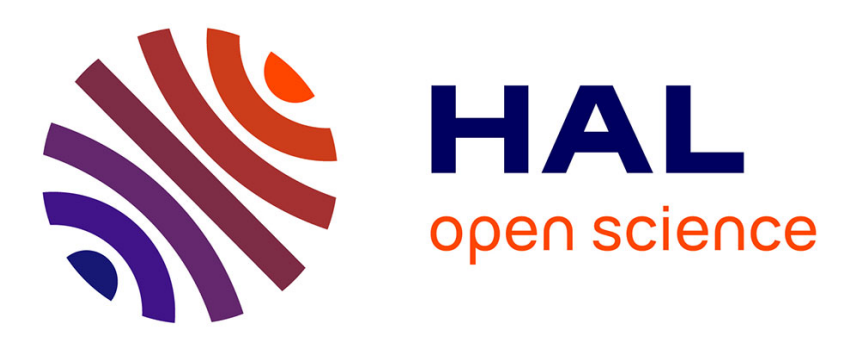

\title{
Espace et stratégies industrielles aux XVIIIe et XIXe siècles : exploiter le laboratoire méditerranéen
}

Gérard Chastagnaret, Olivier Raveux

\section{To cite this version:}

Gérard Chastagnaret, Olivier Raveux. Espace et stratégies industrielles aux XVIIIe et XIXe siècles: exploiter le laboratoire méditerranéen. Revue d'Histoire Moderne et Contemporaine, 2001, 2-3, pp.1124. halshs- 00428540

\section{HAL Id: halshs-00428540 https://shs.hal.science/halshs-00428540}

Submitted on 29 Oct 2009

HAL is a multi-disciplinary open access archive for the deposit and dissemination of scientific research documents, whether they are published or not. The documents may come from teaching and research institutions in France or abroad, or from public or private research centers.
L'archive ouverte pluridisciplinaire HAL, est destinée au dépôt et à la diffusion de documents scientifiques de niveau recherche, publiés ou non, émanant des établissements d'enseignement et de recherche français ou étrangers, des laboratoires publics ou privés. 


\title{
Espace et stratégies industrielles aux $\mathrm{XVIII}^{\mathrm{e}}$ et $\mathrm{XIX}^{\mathrm{e}}$ siècles : exploiter le laboratoire méditerranéen
}

\author{
Gérard Chastagnaret et Olivier Raveux \\ UMR TELEMME, Aix-en-Provence
}

Cet article voudrait, dans une démarche essentiellement programmatique, souligner l'importance de la composante spatiale dans la détermination des stratégies industrielles, dimension qui nous paraît par trop négligée aujourd'hui. Il y a très longtemps que la décision fait partie intégrante du champ de l'analyse économique ${ }^{1}$. Elle y est entrée d'abord sous l'angle, suggestif mais très particulier, de la théorie des jeux : le célèbre théorème de Bernoulli peut servir de repère initial d'une filière intellectuelle qui connaît des moments d'épanouissement, comme les années 1940 et le début des années 50, "cycle" d'intérêt marqué notamment par les travaux de John Von Neumann et Oskar Morgenstern avant de se clore par l'article de Maurice Allais en $1953^{2}$. Les années 1990 ont été marquées par un nouveau rebond, en liaison avec la floraison des pratiques spéculatives et avec un affinement des démarches méthodologiques ${ }^{3}$. L'analyse de la décision économique est aussi au cœur de l'analyse libérale, avec l'arbitrage entrepreneurial après examen des avantages comparatifs, une filière intellectuelle enrichie elle aussi de renouvellements théoriques, à partir du marginalisme. Elle s'est surtout ensuite élargie à d'autres domaines relevant de stratégies d'anticipation, tels que les marchés et la gestion des firmes. Cette diversité des approches, qui pourrait trouver bien d'autres illustrations, n'a pas empêché une convergence certaine des acquis sur la détermination des stratégies entrepreneuriales, dans des domaines aussi variés que la gestion des facteurs de production, les rapports entre les différents acteurs de la vie économique, les marchés ou les contraintes politiques et institutionnelles. Les cadres temporels de la décision ont fait l'objet d'une attention réelle, en dehors même des travaux sur les spéculations boursières ou commerciales et pour des motifs qui ne relèvent pas seulement de la prise en compte traditionnelle du temps dans le coût du capital: la décision est bien perçue comme projection dans le futur,

\footnotetext{
${ }^{1}$ Pour un résumé de l'évolution de l'intérêt des économistes pour la prise de décision, $c f$. Christian SMIDT, "Les théories de la décision et la théorie économique", Les Cahiers Français, n $^{\circ} 280$, mars-avril 1997, p. 49-58.

2 John Von NEUMAN et Oskar MORGENSTERn, Theory of Games and Economic Behavior, Princeton, Princeton University Press, 1944 et Maurice ALLAIS, "Fondements d'une théorie positive des choix comportant un risque et critique des postulats et axiomes de l'Ecole américaine" in Econométrie. Colloques internationaux du CNRS, Paris, vol. 40, 1953, p. 127-140.

${ }^{3}$ Ce renouveau de la théorie des jeux est symbolisé par l'attribution du prix Nobel à John Nash, John Harsanyi et Reinhard Selten en 1994.
} 
anticipation de conditions et de résultats. En revanche, les cadres spatiaux n'ont guère retenu l'attention des chercheurs, sauf dans les cas où les jeux sur l'espace étaient manifestement au cœur du processus décisionnel, notamment dans le cas des spéculations commerciales sur les grains de l'époque pré-industrielle ${ }^{4}$.

Avant d'être, stricto sensu, terrain d'action de l'entreprise, l'espace est pourtant l'une des composantes de base de stratégies qui doivent toujours s'organiser, même implicitement, en fonction d'un espace, et même le plus souvent en fonctions d'espaces différents par leur situation ou par leur échelle. Les jeux d'espace sont loin d'être l'apanage de négociants arbitrant entre les opportunités de marché. L'espace pensé, utilisé ou construit est tout autant au cœur de l'activité industrielle, non seulement pour les aspects commerciaux de l'activité, à l'amont et à l'aval de la production - fourniture des inputs et écoulement des produits - mais aussi pour les stratégies productives elles-mêmes, depuis le choix des sites jusqu'à la mobilisation des facteurs capital et travail.

Champ d'élection du grand négoce de l'époque moderne, la Méditerranée constitue paradoxalement un terrain exceptionnel d'analyse de la composante spatiale des stratégies industrielles. D'abord parce que l'industriel ne doit jamais perdre les réflexes du négociant, pour exploiter les opportunités liées à l'inégalité des espaces, dans le cadre de cycles de durée très variable. Ensuite parce qu'il lui faut savoir jouer de manière spécifique, sans jamais ou presque pouvoir se contenter de se reposer sur les cadres protecteurs de toute industrialisation à ses débuts : l'espace national ou de petits marchés régionaux abrités par les difficultés de transport, et cela pour différentes raisons. Dans le premier cas, depuis les divergences d'intérêt au sein d'un même espace jusqu'à la faiblesse des cadres nationaux, dans le second parce que la mer apporte toujours les contraintes et les chances de l'ouverture. Dans cette perspective, par la diversité des situations qu'elle met en scène comme par ses recompositions spatiales sans cesse renouvelées pour des raisons elles-mêmes en évolution, la Méditerranée des $\mathrm{XVIII}^{\mathrm{e}}$ et $\mathrm{XIX}^{\mathrm{e}}$ siècle est un champ d'études prometteur pour saisir la complexité et la variété des jeux de l'industrie et de ses acteurs sur l'espace. De Séville à Salonique, de l'époque des Lumières aux prémices de la seconde révolution industrielle, l'action des entrepreneurs des rives méditerranéennes peut se lire simplement en termes d'innovation, d'investissement ou de recherche de marchés. Mais, plus qu'ailleurs, ou plus visiblement, leur réussite a dû être aussi décryptage d'espaces encore confus, jeux complexes sur leur croisement et sur leurs échelles. Il ne s'agit pas pour nous de revendiquer une quelconque et fallacieuse originalité méditerranéenne, mais avec sa culture négociante, ses routes de mer et ses

${ }^{4} C f$. notamment, Fernand BRAUDEL, "Choix et stratégies capitalistes" in Civilisation matérielle, économie et capitalisme, $X V^{e}-X V I I I^{e}$ siècle. II : les jeux de l'échange, Paris, Armand Colin, 1979, p. 353-382. 
arrière-pays isolés si proches des côtes, la Méditerranée exacerbe les contrastes, les nécessités et les opportunités. L'industriel ne peut ignorer l'espace. Le constat s'applique à la Méditerranée, mais sa validité n'est-elle pas beaucoup plus générale? Ce caractère conférerait au terrain méditerranéen à la fois un intérêt spécifique et un rôle de révélateur.

\section{Une zone d'ombre de la recherche}

\section{Le privilège du temps}

Le manque d'intérêt pour la composante spatiale des décisions et stratégies industrielles est avant tout le résultat des orientations, des problématiques et des méthodologies de l'histoire économique comme de l'économie. L'histoire économique a depuis longtemps privilégié la réflexion sur la conjugaison des temporalités. A cette fin, elle s'est dotée de toute une batterie d'élaboration et de traitement des séries qui ont centré la recherche sur des cadres territoriaux prédéterminés et oublié l'importance des espaces dans les stratégies individuelles et collectives des acteurs. L'histoire quantitative a besoin d'espaces stables pour ne pas dire " fixes". Elle parvient difficilement à prendre en compte la complexité des articulations des cadres spatiaux et des mouvements fondés sur des facteurs aussi externes à l'entreprise que les opportunités géographiques ou des changements de législation douanière. Elle peine à croiser les racines microéconomiques du comportement des acteurs avec ce qui est son principal objet : l'étude macro-économique des séquences qui définissent l'évolution des activités industrielles sur le long terme.

L'espace comme élément moteur des stratégies entrepreneuriales a également échappé en grande partie à l'analyse des sciences économiques. L'orientation de plus en plus marquée de cette discipline pour la modélisation mathématique l'a conduite à négliger des variables qui ne se prêtaient pas à ce type d'approche. L'espace est ainsi perçu comme une donnée intemporelle ou immatérielle et n'est que trop peu considéré comme un facteur éclairant de la diversité des situations économiques ${ }^{5}$. De même, tout ce qui relève de facteurs mentaux ou culturels a rarement été pris en compte et seulement dans le cadre d'approches frustes, négligeant les avancées d'autres disciplines. Ainsi, la psychologie, pourtant sensible à l'insertion spatiale des individus et aux éléments explicatifs des choix territoriaux, n'a guère été sollicitée par les économistes pour orienter leurs enquêtes vers les anticipations spatiales des agents ${ }^{6}$. Or, le choix

\footnotetext{
${ }^{5}$ Pour cet aspect, $c f$. Patrick VERLEY, "Histoire économique et théorie économique" in Robert BOYER et Yves SAILlARD (dir.), Théorie de la régulation. L'état des savoirs, Paris, La Découverte, 1995, p. 523-526.

${ }^{6}$ Le rapprochement entre la psychologie et l'économie s'est surtout fait sur la question des limites du concept de l'homo aeconomicus. L'entrepreneur n'est pas toujours rationnel, bien
} 
économique relève non seulement d'une forme de rationalité, mais d'un rapport global du décideur à son environnement, rapport qui inclut son histoire personnelle, avec sa formation et, plus largement, l'ensemble de son histoire culturelle mobilisée pour la prise de décision ${ }^{7}$. Une histoire qui a été aussi construction d'un savoir spatial et d'un rapport à l'espace à la fois activés et actualisés par la décision.

\section{L'espace toujours présent}

L'espace est en effet un des éléments essentiels de la prise de décision, parce qu'il intervient dans les représentations - reproductives ou inventives - préalables à la décision et qu'il est l'un des outils ou des enjeux de celle-ci ${ }^{8}$. La décision n'est pas simple enregistrement d'un calcul de probabilité sur les marchés et les profits. La représentation de l'environnement futur sur lequel elle se fonde comporte toujours une composante spatiale et, sous des formes diverses, la décision s'inscrit toujours dans un espace. La crise asiatique est récemment venue rappeler que l'informatique permettait certes d'ignorer les distances et le temps pour les transactions, mais qu'elle ne dispensait nullement d'une attention envers les caractéristiques régionales du "village mondial". Sauf pour les cas, récents à l'échelle de l'historien, des grandes firmes multinationales, des districts “à l'italienne" et des politiques d'aménagement du territoire, les articulations entre stratégies des entrepreneurs et espaces n'ont encore jamais fait l'objet de véritables études alors même qu'elles constituent le plus souvent le socle du dynamisme économique.

La réflexion sur les stratégies spatiales ou, plus exactement, sur la composante spatiale des stratégies élaborées par les acteurs de la vie économique n'est nullement réduction de la décision à l'une de ses composantes : c'est, au contraire, un élargissement du champ d'analyse par la réintégration de l'espace comme composant à part entière du processus décisionnel. Il s'agit d'une approche exigeante, qui suppose la mise en articulation de deux nécessités. Elle appelle d'abord, de la part de l'historien, une connaissance approfondie des logiques propres au secteur étudié et des environnements au sein desquels les stratégies sont pensées et mises en œuvre. Elle demande aussi une collaboration entre plusieurs

informé et calculateur ( $c f$. Christine ROLAND-LEVY et Philippe ADAIR (dir.), Psychologie économique. Théories et applications, Paris, Economica, 1998 et Hervé LAROCHE, "Les décisions stratégiques ou la petite fabrique de la stratégie d'entreprise", Sciences Humaines, $\mathrm{n}^{\circ} 2$, mai-juin 1993, p. 20-23).

${ }^{7}$ Ce point est particulier bien perçu par les historiens modernistes ( $c f$., par exemple, Franco AngIOLINI et Daniel Roche (dir.), Cultures et formations négociantes dans l'Europe moderne, Paris, Editions de l'Ecole des Hautes Etudes en Sciences Sociales, 1995).

${ }^{8}$ Sur l'importance des représentations spatiales, $c f$. l'ouvrage de Marcel RoNCAYOLO, Marseille : port, ville, pôle, Marseille, Chambre de Commerce et d'Industrie de MarseilleProvence, 1990. 
disciplines (histoire, économie, géographie, psychologie, sociologie) qui, sans être neuve sous tous ses aspects, revêtirait néanmoins un caractère pionnier dans plusieurs domaines.

\section{La Méditerranée : un terrain d'observation exceptionnel}

\section{Espaces et secteurs d'activités : le rôle de la longue durée}

Deux types de raisons, tenant d'une part à l'espace et d'autre part au temps, font de la Méditerranée des $\mathrm{XVIII}^{\mathrm{e}}$ et $\mathrm{XIX}^{\mathrm{e}}$ siècles un cadre exceptionnel pour l'analyse spatiale de la décision entrepreneuriale dans le secteur industriel. L'espace méditerranéen est à la fois concentration de diversités et exacerbation de contrastes. La diversité est d'abord celle des secteurs industriels : de la sidérurgie aux secteurs non leaders comme les activités liées à l'agro-alimentaire ou au travail du bois, tous les types d'industries peuvent être recensés ${ }^{9}$. Ce sont aussi de multiples cloisonnements, naturels, politiques, mais aussi culturels et économiques : le simple marché des cartes à jouer en fournit une illustration à la fois symbolique et effective. Les contrastes, ce sont ceux qui opposent les microterritoires, ruraux ou urbains, aux vastes ouvertures, par les ports et des routes commerciales dont les permanences s'appuient sur le renouvellement des produits. Ces contrastes sont aussi ceux des situations locales et sont en l'occurrence souvent aggravés de discordances diachroniques, fondées moins sur des décalages par rapport à une conjoncture dite "générale " que sur des bases spécifiques de prospérités cycliques toujours régionalisées.

Diversité et contrastes mettent l'espace au cœur de la décision, dans le cadre d'arbitrages qui sont rarement sélection et surtout jeux, combinaisons, par des articulations entre espaces disjoints ou entrelacés avec des échelles multiples : la ville et son territoire, le cadre régional, le marché national, un horizon méditerranéen transnational, et souvent même des ouvertures extraméditerranéennes constituent le paysage composite que tout entrepreneur doit savoir lire, dans lequel il doit être capable à tout le moins de se mouvoir sans commettre d'erreur majeure d'appréciation et qu'il doit surtout savoir exploiter et organiser à son profit. Il ne faut pas s'y tromper : au delà de sa complexité, qui est réelle, le champ possède aussi une unité profonde, dans les liaisons entre ces différents espaces, visibles ou sous-jacentes, toujours riches d'opportunités comme de liberté de décision et donc lourdes de responsabilité pour les entrepreneurs. Ces liaisons appellent une attention toute particulière, non seulement parce qu'elles permettent de toucher au cœur des stratégies entrepreneuriales, mais aussi parce qu'elles constituent

9 Gérard Chastagnaret, "L’industrie en Méditerranée : une histoire en construction", Méditerranée, $\mathrm{n}^{\circ}$ 3-4, 1997, p. 7 ; Jordi NADAL et Jordi CATALAN (dir.), La cara oculta de la industrialización española. La modernización de los sectores no líderes (siglos XIX y XX), Madrid, Alianza editorial, 1994. 
une voie exceptionnelle de compréhension des processus même de l'industrialisation en Méditerranée ${ }^{10}$.

En Méditerranée plus qu'en aucun autre lieu, le temps vient aussi nourrir l'espace. Depuis l'Antiquité, la Méditerranée a vu se sédimenter, se transformer ou se renouveler les activités de commerce et de production. $\mathrm{La}$ très longue durée alimente les imaginaires, par les récits et les signes matériels, voire les liens intergénérationnels. De la fin du XVIII ${ }^{\mathrm{e}}$ siècle aux années 1860, les vestiges des activités minières antiques dans la péninsule ibérique ou dans les Balkans sont lus plus souvent comme des appels à de nouvelles exploitations qu'en symboles d'un passé définitivement révolu ${ }^{11}$. Nous l'avons dit: le négoce est plus utilisateur d'héritages maritimes qu'inventeur de nouvelles routes : le trafic entre Marseille et les Echelles du Levant exprime tout à la fois la stabilité des directions et le renouvellement des échanges. Dans cet espace pourtant structurellement voué aux reconversions productives, l'industrie connaît aussi des enracinements pluriséculaires de filières techniques, savoir-faire et débouchés cumulant leurs propres logiques de sédimentation. Cordoue et Marseille restent, aujourd'hui encore, associées l'une au travail du cuir, l'autre à la fabrication des produits chimiques, alors même que ces industries vieilles de plusieurs siècles ont connu de nombreux renouvellements des procédés et des produits.

\section{Espace et industrialisation en Méditerranée : l'intérêt des ruptures}

La longue durée fait plus qu'élargir l'éventail des situations: elle invite à réfléchir, en termes structurels, à la liaison entre caractéristiques de l'économie et traitement des espaces. En ce sens, les périodes de rupture, dont sait de surcroît l'apport documentaire, sont sans doute plus riches d'enseignements que les grandes séquences de l'évolution des activités industrielles et commerciales. Précisément, le basculement de l'économie marchande de l'époque moderne vers l'industrialisation contemporaine constitue l'un des pôles d'intérêt majeur de l'espace méditerranéen. La diversité des rythmes et des modalités de ce passage selon les secteurs et les régions offre des possibilités de comparaison très éclairantes sur le poids respectif de chaque type de contrainte et d'acteur ${ }^{12}$.

\footnotetext{
${ }^{10}$ Patrick VERLEY, L'Echelle du monde. Essai sur l'industrialisation de l'Occident, Paris, Gallimard, 1997, p. 80.

${ }^{11}$ Gérard ChastagnARET, "El Mediterráneo, frontera minera de Europa en el siglo XIX : de la busca del signo al acaparamiento del horizonte próximo" in Industrialización y crecimiento económico en España, Homenaje al Profesor Jordi Nadal, Barcelone, 1999, p. 813-826.

12 Sur ce point, $c f$. Louis BERGERON (dir.), La Croissance régionale dans l'Europe méditerranéenne, $X V I I I^{e}-X X^{e}$ siècles, Paris, Editions de l'Ecole des Hautes Etudes en Sciences Sociales, Paris, 1992.
} 
Les inégalités chronologiques les plus manifestes sont celles des dates mêmes des processus d'industrialisation tout au long du XIX ${ }^{\mathrm{e}}$ siècle. Si la Provence, l'Andalousie, la Catalogne, le royaume des Deux-Siciles et l'Egypte sont touchés par le phénomène tôt dans le siècle, il faut attendre les années 1850-1860 pour que la côte ligure connaisse ses premiers succès et les années 1870-1880 pour que la ville du Pirée s'affiche comme le cœur industriel de la Grèce. Les discordances des temps se retrouvent aussi dans la durée nécessaire à la transformation des structures. La rapidité des changements qui interviennent à Marseille et à Barcelone dans les années 1830-1840 tranche avec la lenteur des transformations dans la grande majorité des régions italiennes et dans le reste de la péninsule ibérique ${ }^{13}$.

Ces écarts dans les temporalités permettent d'étudier les mêmes phénomènes économiques au sein d'environnements multiples. Le problème, classique au point de donner lieu à des réflexions souvent redondantes, du protectionnisme et du libre-échange, retrouve ainsi une nouvelle fraîcheur, à la faveur de la confrontation d'expériences très diverses d'alternance des deux situations, et surtout parce que la Méditerranée invite à l'insérer dans une thématique plus large, celle de la frontière, pertinente sur la très longue durée et aux incidences fortes et originales: les jeux sur la frontière sont partie intégrante des stratégies économiques.

Reste la question même de la rupture induite par le passage vers l'industrie moderne: en quoi les stratégies spatiales sont-elles réellement éclairantes ? La réponse n'est pas dans leur existence même - le commerce traditionnel savait déjà penser et gérer des espaces complexes - mais dans l'apparition d'exigences nouvelles et de réponses spécifiques par rapport à la période antérieure. Le négoce exigeait avant tout une information précoce et sûre, un système de correspondants solides et fiables pour pratiquer avec profit le trafic des grains ou le commerce atlantique ${ }^{14}$. Les solutions consistaient le plus souvent en une combinaison de correspondants locaux et d'utilisation de ressources familiales, notamment de jeunes en cours de formation. Comme l'a montré Charles Carrière, les plus grands négociants voyageaient très peu : le réseau de correspondants suffisait à amener les

\footnotetext{
${ }^{13}$ Xavier DAUMALIN et Marcel CoURduRIE, Vapeur et Révolution industrielle à Marseille (1831-1857), Marseille, Chambre de Commerce et d'Industrie de Marseille-Provence, 1997 ; Olivier Raveux, Marseille, ville des métaux et de la vapeur au XIX ${ }^{e}$ siècle, Paris, Editions du CNRS, 1998 et "Marseille et Barcelone (1831-1848) : contrainte énergétique et industrialisation" in Robert BELOT, Michel COTTE et Pierre LAMARD (dir.), La Technologie au risque de l'histoire.- Paris, Berg internatrional-UTBM, 2000, p. 43-50 ; Jordi NADAL, Jordi MALUQUER DE MOTES et alii, Historia economica de la Catalunya contemporània, segle XIX: Indùstria, transports $i$ finances, Barcelone, Enciclopèdia Catalana, 1991 et Catalunya, la fàbrica d'Espanya. Un siglo de industrialización catalana, 1833-1936, Barcelone, Ayuntamiento de Barcelona, 1985.

${ }^{14} \mathrm{C} f$. Gilbert BUTI, "Marseille au XVIII ${ }^{\mathrm{e}}$ siècle : réseaux d'un port mondial" in Michèle ColLIN, Ville et port. XVIII'-XX siècles, Paris, l'Harmattan, 1994, p. 209-222.
} 
affaires et à assurer la sécurité des transactions ${ }^{15}$. L'industrialisation ne fait pas disparaître les besoins du négoce, mais elle y ajoute de nouvelles exigences. Certaines restent de caractère commercial comme l'utilisation et l'exploitation de nouvelles matières premières ou l'application d'innovations dans le domaine des transports. D'autres, liées à l'implantation des activités productives, impliquent de nouveaux rapports à l'espace, du fait de la diversification des sites et parfois de la nature des activités. Ces exigences concernent l'ensemble du processus décisionnel, depuis la définition de la stratégie jusqu'à la gestion des nouvelles implantations. Le cas extrême est sans doute celui des activités à effectuer dans le cadre de concessions publiques et à forts effectifs ouvriers, comme les concessions de services publics et surtout de mines : le "démarchage" initial à l'égard de la puissance publique doit s'accompagner d'une vigilance dans la longue durée. Les péripéties des initiatives françaises en Grèce dans le Laurium, au cours des années 1860-1870, illustrent les difficultés de ce type de situation. Il faut aussi installer une unité productive, avec toutes les implications, techniques et humaines, de l'opération. Ce dernier type d'exigence vaut pour toute implantation industrielle, toujours confrontée à des problèmes d'insertion, dans un site et dans un ensemble social, ainsi qu'aux aspects relevant de facteurs technologiques ou de l'organisation du travail $^{16}$. Ces nouvelles formes de rapport à l'espace ne peuvent être mises en place ou gérées dans le seul cadre des pratiques traditionnelles du mandataire commercial ou du simple correspondant. Elles entraînent de nouvelles responsabilités pour les entrepreneurs impliquant notamment des formes de présence soutenue sur le terrain des opérations ${ }^{17}$.

\section{Trois approches convergentes}

Vaste et complexe, le problème des stratégies économiques et des espaces en Méditerranée se doit d'être abordé à partir de trois thèmes forts qui constituent des angles d'attaque convergents : l'espace perçu par les agents économiques comme élément fondateur de décisions et de stratégies ; l'espace structuré et contrôlé par les activités entrepreneuriales ; l'espace transformé par des stratégies industrielles et commerciales lourdes d'implications économiques mais aussi politiques et sociales. Articulés d'amont en aval, ces trois points ne sont que des cadres de réflexion et ne constituent en rien une segmentation de la problématique. Dans la conduite

\footnotetext{
${ }^{15}$ Charles CARrIERE, Négociants marseillais au XVIII ${ }^{e}$ siècle. Contribution à l'étude des économies maritimes, Marseille, Institut Historique de Provence, 1973, 2 volumes.

${ }^{16}$ Sur ces deux points, $c f$. B. L. ANDERSON et P. W. PILling, "Spanish Entrepreneurs and British Technology in Early XIXth Century Andalucia", Journal of European Economic History, 1990, vol. 19, 1, p. 35-72.

${ }^{17}$ Gérard Chastagnaret, "La Méditerranée au temps de l'industrialisation" in Thierry FABRE (dir.), Rencontres d'Averroès. La Méditerranée, frontières et passages, Arles, Actes Sud, 1999, p. 143.
} 
de ses travaux, le chercheur doit réunir ces trois éléments au sein d'une analyse générale qui est la seule garantie de restitution de la richesse et de la complexité du lien entre stratégies économiques et espaces.

\section{L'espace perçu : genèse et maturation de la prise de décision}

Il s'agit d'abord d'inventorier les éléments qui permettent aux agents de l'économie de penser l'espace et de comprendre comment ceux-ci s'articulent pour motiver et expliquer la prise de décision industrielle ou commerciale. Les recherches doivent avoir pour but de proposer des grilles de lecture de ces différents mécanismes, notamment dans les deux grands types de résolution que prennent les entrepreneurs dans la gestion de leurs affaires : les anticipations qui inaugurent des cycles de durée très variable au sein d'espaces qu'il reste à structurer et les adaptations qui procèdent de modifications conjoncturelles ou structurelles s'opérant au sein d'ensembles spatiaux déjà constitués. Les anticipations "fondatrices" constituent, de loin, l'enjeu majeur pour la réflexion sur l'espace, parce qu'elles posent une question majeure : qu'est-ce qu'inventer l'espace ? S'agit-il d'effectuer une projection originale, fondée sur une analyse prévisionnelle rationnelle des différents paramètres, de transposer des modèles à partir d'autres espaces ou d'autres époques, de mettre en œuvre des imaginaires par l'actualisation de signes ? Dans la réalité, les alternatives sont sans nul doute beaucoup moins nettes, mais il n'en demeure pas moins que l'invention d'un espace à venir s'exerce au sein de deux polarisations majeures dans l'élaboration des représentations, d'une part entre le conservatisme et l'inventivité, d'autre part entre le rationnel et l'incantatoire. L'espace est un révélateur d'attitudes profondes à l'égard du futur.

Dans cette perspective, le croisement de l'espace et de l'innovation est particulièrement éclairant, par la typologie, contrastée à l'extrême, qu'il permet de dégager au sein des pratiques des industriels de la Méditerranée. L'espace peut servir d'abord à refuser ou à masquer le temps, lorsqu'on lui fait jouer le rôle de back ground figé au sein duquel les agents économiques cherchent à minimiser les risques en se limitant à reproduire des activités et des attitudes validées par la durée. A Marseille, entre la fin du XVII ${ }^{\mathrm{e}}$ siècle et les débuts de la monarchie de Juillet, l'indiennage et la savonnerie conservent les mêmes caractéristiques techniques parce que leur positionnement sur des créneaux commerciaux spécifiques n'impose aucune nécessité de modifier les structures et l'organisation de la production. Dans d'autres cas, cette segmentation de l'espace peut avoir une fonction d'incubateur d'initiatives fondatrices, liées à des spécificités locales. L'invention du four à manche (horno de gran tiro), en 1846, par un pharmacien de Carthagène pour traiter les carbonates de plomb locaux, est une réponse à un blocage qui ne peut être surmonté par l'importation de 
procédés étrangers ${ }^{18}$. L'espace segmenté peut aussi être l'instrument d'une inscription dans la durée en constituant le cadre de ces renouvellements sectoriels cycliques, caractéristiques de plusieurs sites méditerranéens : la meilleure illustration en est peut-être celle d'Hermoupolis, capitale des Cyclades, qui, de 1850 au milieu du $\mathrm{XX}^{\mathrm{e}}$ siècle, offre une palette d'industries étonnante par sa diversité et sa capacité de recomposition. L'innovation en Méditerranée est aussi un lieu privilégié d'analyse des stratégies de transfert, par introduction de productions ou de pratiques commerciales déjà éprouvées dans un autre ensemble territorial et surtout par adoption d'innovations. Ce dernier mouvement est moins unilatéral qu'on ne le croit souvent. La Méditerranée est aussi espace "naisseur" d'innovations. Ainsi, l'Italie est au début du XVII ${ }^{\mathrm{e}}$ siècle un des pôles de référence en Europe occidentale dans l'industrie de la soie, au point de susciter l'intérêt de nombreuses nations qui viennent y chercher techniciens et équipement. L'entreprise de l'Anglais John Lombe, qui installe en 1717 près de Derby un moulin à organsiner avec des machines réalisées sur des plans rapportés d'Italie, constituent un exemple des plus significatifs ${ }^{19}$. Des sites peuvent en venir à s'identifier à une technique. C'est le cas de Murano, dont les verriers conservent pendant longtemps le secret de la fabrication des miroirs de grande taille ; c'est aussi celui de Marseille, à partir des années 1830, pour la fabrication des presses hydrauliques de traitement des oléagineux ${ }^{20}$. Les mouvements s'exercent aussi très largement, et de plus en plus au cours du XIX ${ }^{\mathrm{e}}$ siècle, en direction de la Méditerranée, mais deux observations s'imposent. Tout d'abord, ils sont beaucoup moins signe de dépendance que d'appropriation dynamique, l'installation d'ingénieurs britanniques de premier plan s'inscrit dans cette ambivalence de l'Espagne. Par ailleurs, les transferts d'innovations sont beaucoup plus complexes qu'un simple courant d'importation de techniques nouvelles envers un espace retardataire ou déprimé : les phénomènes intra-méditerranéens jouent un rôle important, voire parfois de premier plan, illustrant, au delà des retards relatifs au sein d'un même ensemble, l'élaboration de modèles et la mise en place de dynamiques régionales. L'espace n'est donc pas neutre : il pèse sur les acteurs et il peut être subi ou instrumentalisé par eux, de manière très inégalement heureuse mais toujours significative, au service de conservatismes ou de volontés innovantes.

\section{L'espace structuré : constructions et jeux d'opportunité}

\footnotetext{
${ }^{18}$ Cf. Gérard CHASTAGNARET, L'Espagne puissance minière dans l'Europe du XIX $X^{e}$ siècle, Madrid, Casa de Velázquez, 2000.

${ }^{19}$ Patrick VERLEY, Entreprises et entrepreneurs du XVIII siècle au début du XX siècle, Paris, Hachette, 1994, p. 21.

${ }^{20}$ Ibidem, p. 22 et Olivier RAVEUX, Marseille..., op. cit., p. 149-150.
} 
L'industriel ne doit pas seulement inscrire sa stratégie dans un espace, il doit aussi gérer celui-ci et parfois même le construire. Il convient en effet de distinguer les opérations de création d'espaces économiques de celles relevant de la gestion d'ensembles préalablement structurés et déjà opérationnels. La réussite d'un projet industriel ou commercial et son inscription dans la durée découlent fondamentalement de la capacité des entrepreneurs à combiner, dans le même temps, la mise en place de dynamiques internes à l'activité économique et la maîtrise des contraintes de l'environnement, liées à la conjoncture ou aux cadres mêmes de l'activité. L'espace méditerranéen invite à une attention privilégiée dans trois directions.

La première est celle des stratégies de frontières ou, plus largement, de jeu sur des espaces de statuts différents. On peut observer, selon les cas, une grande diversité de pratiques, d'ailleurs parfois associées : acceptation de la règle du cadre économique national, combinaison d'espaces d'échelles différenciées (le local, le régional, le national et le supranational), jeux sur l'inégalité des espaces dans la conquête des marchés, pratiques de contrebande, dont on sait qu'elles constituent souvent, de par leur risque même, un moyen de dégager d'importants profits. L'inscription des comportements dans cet éventail, qui pourrait sans nul doute être détaillé et élargi, appelle un décryptage décisif pour la compréhension globale du projet de l'entreprise.

La seconde direction de réflexion concerne les variables, productives, commerciales, ou "environnementales", privilégiées par les entrepreneurs : capitaux, main-d'œuvre, technologie, moyens de transport, marchés et relations avec les institutions. Tout au long du XIX ${ }^{\mathrm{e}}$ siècle, les industriels et les négociants cherchent souvent à se constituer en groupes de pression auprès des pouvoirs publics pour verrouiller ou contrôler un espace à leur profit. Les industriels espagnols du textile et la sidérurgie ont su obtenir une protection douanière pour se réserver le marché national. Cette stratégie peut même prendre la forme d'une demande d'intervention militaire ou d'occupation effective d'un territoire. Le cas de Marseille avec l'Algérie et l'Ouest africain dans les années 1830-1840 est significatif de ce type de pratiques ${ }^{21}$. De ce fait, l'analyse de la perception de l'espace et des projets dont il est porteur est indissociable d'une étude des réseaux du pouvoir économique. Ce phénomène prend une ampleur particulière lorsque l'entreprise opère au sein d'espaces de statuts différents. Le recours à des réseaux politiques, familiaux, culturels ou confessionnels est alors souvent le moyen le plus fréquent pour vaincre la complexité d'un fonctionnement qui nécessite accommodations et adaptations face à des nécessités difficilement compatibles et parfois diamétralement opposées. On sait déjà le rôle déterminant de ces réseaux dans la maitrise des marchés, on le

${ }^{21}$ Cf. Xavier DAUMALIN, Marseille et l'Ouest africain : l'outre-mer des industriels (18411956), Marseille, Chambre de Commerce et d'Industrie de Marseille-Provence, 1992. 
connaît moins pour l'acquisition et la diffusion des technologies alors qu'il y est souvent déterminant. Les logiques de l'industrialisation de la métallurgie amènent ainsi les entrepreneurs méditerranéens à activer des réseaux de toute nature pour attirer des hommes porteurs de compétences nord-européennes. La Catalogne, Marseille et Naples accueillent de nombreux techniciens britanniques au cours des années 1820-1840 pour assurer la mise en place de productions à forte valeur technologique comme la construction de bateaux à vapeur, la fabrication des locomotives et le traitement des plombs argentifères ${ }^{22}$. Ces réseaux ne sont pas uniquement organisés sur un axe qui relie Nord et Sud de l'Europe. A l'intérieur même de la Méditerranée, les relations entre entrepreneurs ont permis une diffusion de techniques industrielles de pointe. L'apparition de l'indiennage à Barcelone en 1736 se fonde sur les liens du port catalan avec Marseille ${ }^{23}$. Dans les années 1860 et 1870 , la création des mines et fonderies modernes de plomb du Laurium doit beaucoup à un transfert de technologie depuis la ville espagnole de Carthagène, ainsi d'ailleurs qu'au transfert vers la Grèce de plusieurs centaines d'ouvriers espagnols spécialisés ${ }^{24}$.

Reste enfin la question, fondamentale pour toute activité productive, de la présence in situ d'une vraie capacité de décision. Ce problème est plus important pour l'industrie que pour la plantation, parce que le processus productif est beaucoup plus porteur d'imprévus, demandeur d'adaptations ou de contacts locaux. Surtout en l'absence de téléphone, l'ingénieur directeur n'est qu'une solution partielle à ce problème, d'autant plus que les responsables d'entreprise tiennent à bien marquer la différence entre la compétence technique et la fonction stratégique. La panoplie des réponses apportées - voyages, jeux de communication sur la présence, recherche d'autres soi-même au sein de la famille, contrôle tatillon du directeur local par courrier ou télégraphe - constituent une information précieuse, non seulement sur les méthodes de gestion de la société et les pouvoirs au sein de celle-ci, mais aussi sur ses modalités d'intégration de l'espace dans l'ensemble de sa stratégie. La prise en compte des enjeux spatiaux est lourde d'implications, y compris hors de l'entreprise : la nécessité de la présence, l'intérêt de la création d'un lien de familiarité avec les interlocuteurs

\footnotetext{
${ }^{22}$ M. J. Alvarez PANTOJA, "Nathan Wetherell, un industrial inglès en la Sevilla del antiguo régimen", Moneda y Credito, n 143, 1977, p. 133-186 ; Olivier RAVEUX, "El papel de los técnicos ingleses en la industria metalúrgica y mecánica del norte del Mediterráneo (1835-1875) : una primera aproximación", Revista de Historia Industrial, n 6, 1994-1995, p. 143-161 et "Un technicien britannique en Europe méridionale : Philip Taylor (17861870) ", Histoire, Economie et Société, 2000, n 2 : p. 253-266 ; Luigi DE RosA, Iniziativa e capitale straniero nell'industria metalmeccanica del Mezzogiorno, 1840-1904, Naples, 1698.

${ }^{23}$ James K. J. Thomson, A Distinctive Industrialization : cotton in Barcelona, Cambridge, Cambridge University Press, 1992, p. 74-75.

${ }^{24}$ Christine Agriantoni, Les Débuts de l'industrialisation en Grèce (les années 18701880), Thèse de doctorat, Paris X-Nanterre, 1984.
} 
extérieurs ne sont-ils pas parmi les racines de la pérennité du caractère familial de la gestion des entreprises - autochtones et parfois même étrangères - en Méditerranée ?

\section{L'espace transformé : une relation réciproque}

Le dernier thème de questionnement se situe en aval de l'action des entrepreneurs : quelles sont les retombées des initiatives des industriels et des commerçants sur les espaces eux-mêmes ? Comment des dynamiques industrielles ou commerciales participent-elles à la structuration d'un espace économique collectif, vécu par les populations et les cadres institutionnels ? Ces questions appellent des déclinaisons plus concrètes, comme celle-ci : quels impacts faut-il attribuer aux initiatives entrepreneuriales - de nature individuelle ou collective - dans les processus de formation et de consolidation des états nationaux ? Sous le Premier Empire, les industriels de la soude amorcent la pleine intégration de l'économie marseillaise dans l'espace national français. Dès les années 1840, Marseille n'est plus la "porte de l'Orient" pour son unique profit. Elle est désormais un des liens économiques essentiels entre Europe du nord-ouest et Méditerranée, la tête de pont de la France vers les espaces lointains.

Ces mêmes initiatives des entrepreneurs engendrent également des flux de populations aux niveaux intra et extra méditerranéens. Ces vagues migratoires sont d'autant plus importantes qu'elles permettent de comprimer les coûts salariaux. Marseille a excellé en ce domaine. Les entrepreneurs profitent de la dynamique commerciale du port, d'un monde de correspondants disséminés sur le pourtour méditerranéen depuis la fin du XVII ${ }^{\mathrm{e}}$ siècle pour activer les flux migratoires nécessaires au développement des industries. Les vagues d'ouvriers levantins, italiens et espagnols se succèdent pour venir étoffer des secteurs aussi variés que la faïence, l'indiennage, la soierie, le raffinage du sucre, l'industrie des oléagineux et celle des non-ferreux ${ }^{25}$.

Les initiatives économiques ne font pas que s'inscrire dans un cadre territorial déterminé : elles influencent aussi celui-ci en provoquant des modifications d'équilibre. L'influence réciproque est en fait permanente : l'espace transforme les initiatives aussi bien dans leurs formes que dans leur dessein. Il contribue à remodeler constamment les horizons de la décision et impose sa marque dans l'émergence de nouvelles stratégies et de nouvelles initiatives. En fait, le chercheur ne doit pas analyser l'évolution de l'entreprise seulement en termes de réponse à des évolutions externes : il doit aussi s'attacher à décrypter les malentendus initiaux, les contraintes internes, liées à la technique, aux facteurs de production ou à la gestion, qui peuvent obliger à des révisions de perspectives, voire à des renoncements.

${ }^{25}$ Renée LOPEZ et Emile TEMIME, Migrance. Histoire des migrations à Marseille, $t .1$ et 2 , Marseille, Edisud, 1990. 
Les illusions initiales des entreprises belges établies dans les Asturies au cours des années 1830 trouvent deux solutions très inégales, l'une très médiocre de repli sur un marché houiller espagnol insignifiant, l'autre plus rémunératrice, mais longue à se dessiner, de réorientation vers la filière du $z^{2}{ }^{26}$. L'échec d'une anticipation spatiale fondée sur l'application à la Méditerranée du modèle de consommation de l'Europe du Nord trouve une issue dans une autre construction spatiale : l'ancrage de l'Espagne sur la demande urbaine de l'Europe du nord-ouest. L'exemple illustre aussi que toute reconversion appelle la mobilisation d'un nouvel environnement culturel et qu'elle ne va pas sans lourdes difficultés : la création d'un espace, ou de plusieurs espaces puisque, en l'occurrence, la société finit par créer un marché espagnol du zinc, a besoin de temps. L'espace et le temps peuvent se fortifier ou se détruire réciproquement.

Une réflexion sur les espaces perçus, organisés et transformés par les entrepreneurs de l'industrie dans la Méditerranée des $\mathrm{XVIII}^{\mathrm{e}}$ et $\mathrm{XIX}^{\mathrm{e}}$ siècles poursuit toujours un double objectif. Le premier est propre au champ considéré : il s'agit d'abord de comprendre les modalités de constitution de la représentation de l'espace par les acteurs de l'économie et de parvenir si possible à la construction d'une typologie. En ce domaine, la Méditerranée apporte multiplicité des formes et des articulations. Le second objectif est d'approfondir, pour l'ensemble du processus décisionnel, la fonction et l'impact d'un facteur essentiel et sous-estimé du dynamisme des activités industrielles. L'enjeu est essentiel pour la Méditerranée: on ne peut comprendre les logiques industrielles de la rive Sud de l'Europe sans intégrer les stratégies spatiales de ses acteurs. L'espace au cour de la décision : une marginalité ou une anticipation méditerranéennes ?

\footnotetext{
${ }^{26}$ Ces compagnies ont participé en fait d'une triple illusion, sur la qualité de la houille asturienne, sur le développement des marchés espagnol et méditerranéen, sur la possibilité de concurrencer la houille anglaise en Méditerranée ( $c f$ Gérard CHASTAGNARET, "Une réussite dans l'exploitation des minerais non ferreux espagnols au XIX siècle: la Compagnie Royale Asturienne des Mines" in Aux origines du retard économique de l'Espagne, XVI ${ }^{e}$-XIX $X^{e}$ siècles, Paris, Editions du CNRS, 1983, p. 81-113, et L'Espagne, puissance minière..., op. cit.).
} 